\title{
A Rare Primary Lung Tumor: Pulmonary Epithelioid Hemangioendothelioma and a Literature Review
}

\author{
(1) Hülya DiROL,' 1 Ömer ÖZBUDAK,' (i) İrem Hicran ÖZBUDAK² \\ 'Department of Chest Diseases, Akdeniz University, Antalya-Turkey \\ ${ }^{2}$ Department of Pathology, Akdeniz University, Antalya-Turkey
}

\begin{abstract}
SUMMARY
Epithelioid hemangioendothelioma is an extremely rare vascular tumor, with a prevalence of less than one in one million each year. It is most commonly described in the veins of the extremities, but it may also arise from the vascular endothelial or pre-endothelial cells of the lung. Most of the patients are young women. The clinical behavior of pulmonary endotheloid hemangioma (PEH) ranges from welldifferentiated hemangioma to high-grade angiosarcoma. Chest CT is characterized by the presence of a nodule that is either single or multiple in one lung or both. For the diagnosis, a good histopathological evaluation of the biopsy is required. Depending on the rarity of the disease, there is no standardization in treatment. Chemotherapy can be administered for widespread disease, with unclear benefits. Drugs that block the vascular endothelial growth factor are expected to be effective with a small number of promising case reports. What we know about the disease and how to manage is all those derived from approximately 250 case reports. Thus, we aimed to present one more patient with bilateral pulmonary epithelioid hemangioma with two years follow up results after some chemotherapy.

Keywords: Pulmonary epitheloid hemangioendothelioma; vascular endothelial growth factor-A (VEGF-A), weibelpalade body.

Copyright $\odot 2020$, Turkish Society for Radiation Oncology
\end{abstract}

\section{Introduction}

Epithelioid hemangioendothelioma is an extremely rare vascular tumor, with a prevalence of less than one in one million each year,[1] which arises from the intima of the blood vessels. At the beginning, it was considered as a kind of bronchoalveolar cell carcinoma with a remarkable tendency to invade adjacent blood vessels and small airways. Hence, it was called as an intra-vascular bronchioloalveolar tumor. Later, Weiss and Enzinger identified that it was a borderline soft-tissue vascular tumor between hemangioma and angiosarcoma.[2] Nowadays, it is categorized as a locally aggressive tumor with metastatic potential.[3]
Pulmonary epithelioid hemangioma (PEH) comprises about $12 \%$ of all epithelioid hemangioendothelioma.[1] The majority of the patients are women and about half are younger than 40 years. Most of the patients are asymptomatic and usually diagnosed incidentally on the basis of abnormal chest radiography. As it is a rare disease, there is no standard for the treatment. For the resectable ones, wide local excision with possible regional lymph node dissection is recommended. [4] However, some authors prefer follow up with chest CT at least once a year in asymptomatic patients, evidencebased on the two cases reported $\geq 10$-year survival without therapy.[5,6] Due to the slow growth of the tumor, radiotherapy is ineffective and only preferred for good local control after surgical excision. Chemotherapy 
can be administered for widespread disease, with unclear benefits. Drugs that block the vascular endothelial growth factor are expected to be effective with a small number of promising case reports.[7] What we know about the disease and how to manage is all those derived from approximately 250 case reports. Thus, we aimed to present one more patient with bilateral pulmonary epithelioid hemangioma with two years follow up results after some chemotherapy.

\section{Case Report}

A sixty-year-old female patient admitted to our out-patient clinic with incidental chest CT findings. The patient was evaluated at another center for hoarseness, and she was referred with the suspicion of metastasis upon detection of nodules in the thorax CT. She had no respiratory symptom other than dyspnea that was associated with heart failure before. She was taking cardiac medication, and out of this, her medical history was unremarkable, with no occupational and environmental exposure, no pet at home, and no recent travel history. She never smoked. In chest CT, bilateral multiple nodules, the largest of which was hilar and $2.1 \times 3 \mathrm{~cm}$ in diameter in the right lung upper lobe, had been detected (Fig. 1). In PET CT, all nodules in the lungs were nonmetabolic except that the largest one with low metabolic activity (SUVmax:3.8) (Fig. 2). There were multiple, hypodense nodules with low metabolic activity (SUVmax: 5.1) also in the liver. After the bronchoscopic evaluation and percutaneous liver biopsy were nondiagnostic, a surgical biopsy from lung nodules was performed. Histopathological examination of wedge biopsy from two nodules in the right middle lobe revealed that it was a Pulmonary Epithe-

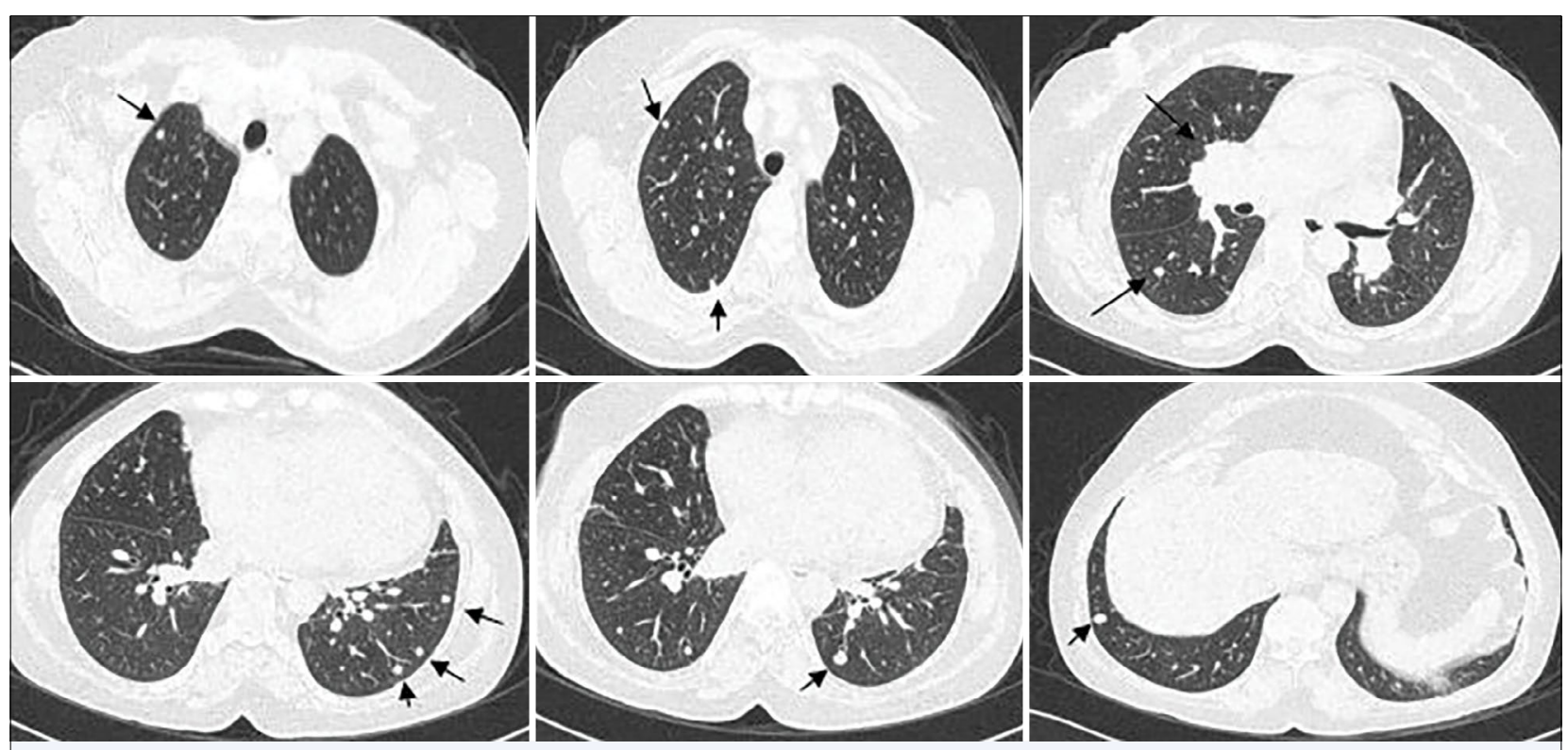

Fig. 1. Chest CT; Bilateral multiple nodules. The largest of them is hilar and $2.1 \times 3 \mathrm{~cm}$ in diameter in the right lung upper lobe.
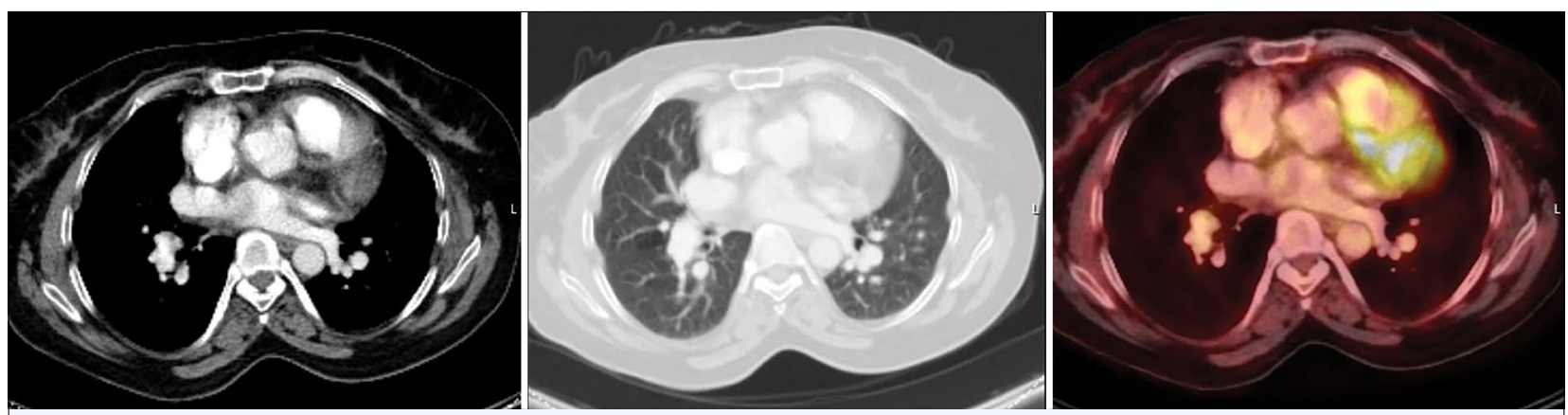

Fig. 2. PET CT; All the nodules are nonmetabolic except the largest one, with low metabolic activity (SUVmax:3.8). 


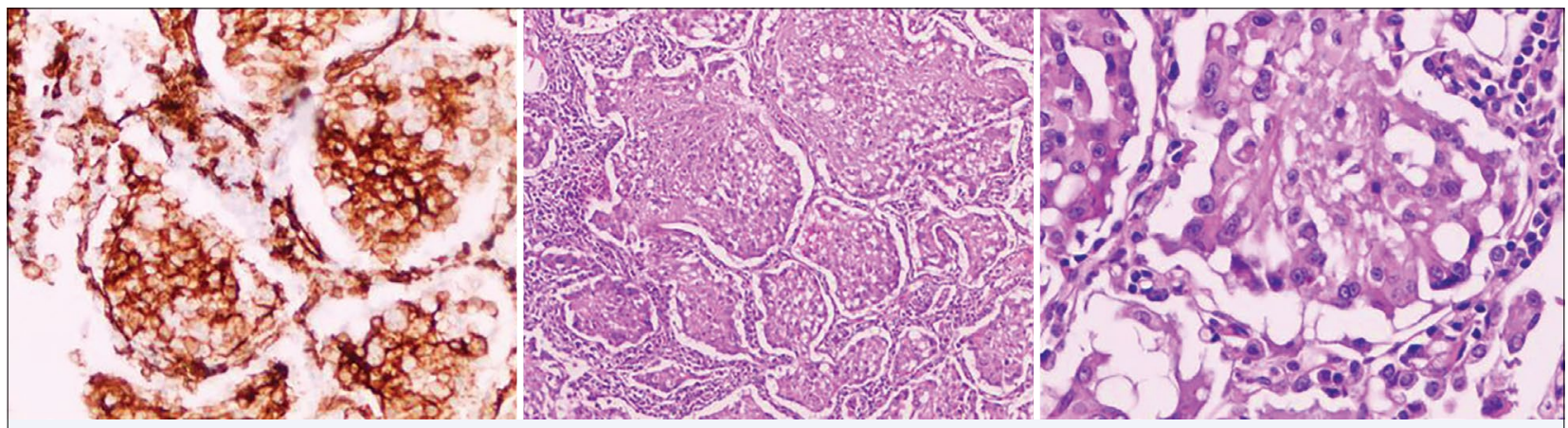

Fig. 3. Immunohistochemically, the tumor cells are positive for CD31. (x200). Epithelioid hemangioendothelioma. The spindle and round-shaped tumor cells are forming nodular lesion by extending to adjacent alveoli through the lumen of respiratory bronchioles which preserve the alveolar frame work. (H\&E, x100). Some round-shaped tumor cells have cytoplasmic vacuoles and erythrocytes or fibrin are identified within these intracytoplasmic lumina. (H\&E, x400).
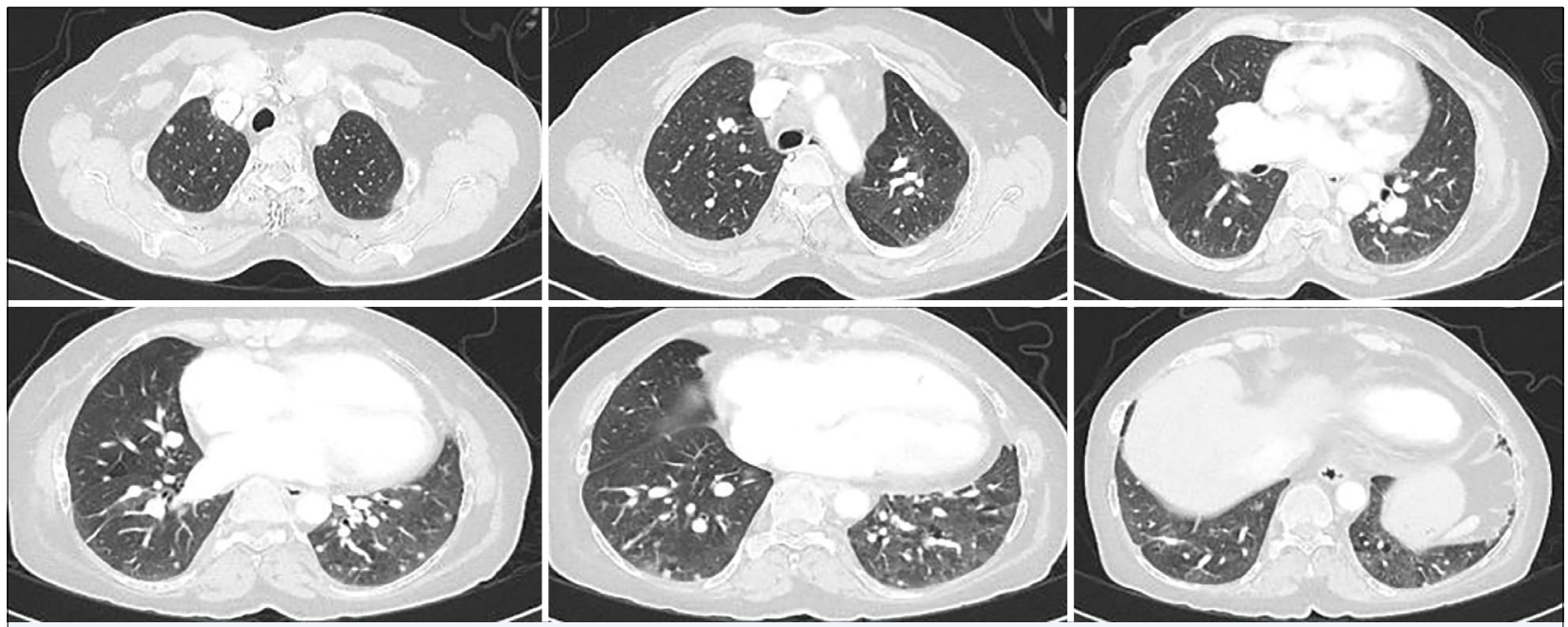

Fig. 4. Some of the nodules disappeared while some were stabile.

lioid Hemangioma (Fig. 3). The patient was started on gemcitabine and docetaxel chemotherapy. After three cycles, radiological regression was accepted as a good response, and chemotherapy was continued. After six cycles, there was progression in pet CT and chemotherapy was changes as cisplatin and Adriamycin. The patient was stable after six cycles and is fallowed without any further chemotherapy every three months for about one year (Fig. 4).

\section{Discussion}

Epithelioid hemangioendothelioma is a moderate malignant vascular tumor, which arises from vascular endothelial or pre-endothelial cells. It may affect multiple organs but most commonly described in the veins of the extremities.[2] The other organs from which this tumor typically arises are the liver, bone, soft tissue and the lung. Multi-organ involvement is also possible at the same time, but single organ involvement is more common. Most of the patients are younger than 40 years of age, the median age of onset is 36 years (range, 20 to 60 years) and almost never seen in childhood.[8-10] No association with a specific etiologic agent other than oral contraceptives for only epithelioid hemangioendothelioma in the liver has been determined.[11] The majority of the patients are women. This raises the suspicion that female hormones may be effective in the development of the disease, as in oral contraceptives.

The clinical behavior of PEH ranges from well-differentiated hemangioma to high-grade angiosarcoma. The clinical spectrum of the disease varies from silent to an aggressive disease with widespread metastasis. 
More than half of the patients are asymptomatic that mostly diagnosed incidentally. Coughing, shortness of breath, pleuritic chest pain and/or hemoptysis is some of the possible respiratory symptoms.[12] Chest CT is characterized by the presence of a nodule that is either single or multiple in one lung or both. Nodules are $1-2 \mathrm{~cm}$ in diameter with margins ranging from well to poor and generally adjacent to small and mediumsized vessels and bronchi.[2] Lymph node metastases, pleural thickening, effusion and ground-glass opacities are other possible radiological findings. PEH is often confused with lung cancer, metastasis or granulomatous diseases.

For the diagnosis, a good histopathological evaluation of the biopsy is required. It is like a hesitation at the primitive stage of vascular differentiation, with undeveloped vascular spaces from endothelial cells in epithelioid or histiocytoid appearance. It should be differentiated from various sarcomas with epithelioid appearance. The other differential diagnoses are melanomas and metastatic carcinomas. Electron microscopic evaluation and immunohistochemical evaluation is useful for the diagnosis. Electron microscopy shows a well-developed basal lamina, picnotic vesicles, and more specific Weibel-Palade bodies. Immunohistochemically, Factor VIII and CD31 antigens are positive in tumor cells of PEH. To understand if the tumor is vascular in origin, CD34 is useful, as this antigen is a sensitive marker of vascular tumors. The other auxiliary markers, HMB- 45 and melanin, can be used for the diagnosis of melanoma. Mitotic activity and nuclear atypia are expected to be higher in carcinoma, melanoma and epithelioid angiosarcoma than in PEH. Necrosis is common and vascular differentiation occurs as the formation of irregular sinusoidal vascular channels in epithelioid angiosarcoma. Sometimes, increased mitotic activity (more than one mitosis at 10 magnification), focal spindle cells and necrosis are present in $\mathrm{PEH}$. In these cases, $\mathrm{PEH}$ exhibits more aggressive behavior.

Depending on the rarity of the disease, there is no standardization in treatment. Wide local excision with possible regional lymph node dissection is recommended for the resectable ones. The mean five-year survival is about $60 \%$ after surgical excision.[13] As the rates of local recurrence are about 10-15\%, some studies recommend radiotherapy fallowing surgery, especially for the ones in bones. However, radiotherapy alone is not appropriate as it is not effective in slow-growing tumors. Surgery can be possible in only cases with unilateral disease with single and/or multiple nodules. If it is bilateral, surgery is impossible and in that situation, chemotherapy can be used with unclear benefits. Till now, Interferon- $\alpha$, Carboplatin, Paclitaxel and Bevacizumab have been tried.[14] The new human vascular endothelial growth factor-A (VEGF-A)-targeting chemotherapeutic agents, bevacizumab and sorafenib, are expected to be effective, with some promising results. $[7,15]$ In general, tumor is slowly progressive and survival of 10 years or more without therapy has been reported before. [5,6] Considering these results, observation may also be an option for asymptomatic $\mathrm{PEH}$ patients, and start chemotherapy when the patient becomes symptomatic or lesions progressively enlarge.

\section{Conclusion}

In conclusion, epithelioid hemangioendothelioma, which is a rare primary lung tumor, may exhibit a clinical behavior ranging from high-grade angiosarcoma to well-differentiated hemangioma, and we try to treat these patients properly with the expectation of an effective and standardized treatment method as soon as possible.

Informed consent: Written informed consent was obtained from the patient for the publication of the case report.

Peer-review: Externally peer-reviewed.

Conflict of Interest: No conflict of interest to declare.

Financial Disclosure: No funding was received.

Authorship contributions: Concept - H.D., Ö.Ö., İ.Ö.; Design - H.D.; Supervision - H.D., Ö.Ö., İ.Ö.; Materials - H.D., İ.Ö.; Data collection \&/or processing - H.D.; Analysis and/ or interpretation - H.D., Ö.Ö., İ.Ö.; Literature search - H.D.; Writing - H.D.; Critical review - H.D., Ö.Ö., İ.Ö.

\section{References}

1. Lau K, Massad M, Pollak C, Rubin C, Yeh J, Wang J, et al. Clinical patterns and outcome in epithelioid hemangioendothelioma with or without pulmonary involvement: insights from an internet registry in the study of a rare cancer. Chest 2011;140(5):1312-8.

2. Weiss SW, Enzinger FM. Epithelioid hemangioendothelioma: a vascular tumor often mistaken for a carcinoma. Cancer 1982;50(5):970-81.

3. World Health Organization (WHO); International Association for Research on Cancer: In World Health Organization Classification of Tumours: Pathology and Genetics of Tumours of Soft Tissue and Bone. Fletcher CDM, Unni KK, Mertens F, editors. Lyon, France: IARC Press; 2002. Available at: https://aaot. org.ar/_docs/pdfs/who-classification.pdf. Accessed Mar 12, 2020. 
4. Scordi-Bello IA, Snyder A, Schwartz M, Fallon JT. Intravascular epithelioid hemangioendothelioma of the inferior vena cava: case report of an unusual and unpredictable vascular tumor. Cardiovasc Pathol 2009;18(4):243-6.

5. Okamura K, Ohshima T, Nakano $R$, Ouchi $H$, Takayama K, Nakanishi Y. A case of pulmonary epithelioid hemangioendothelioma surviving 10 years without treatment. Ann Thorac Cardiovasc Surg 2010;16(6):432-5.

6. Fujita K. Long term follow-up of a case of pulmonary epithelioid hemangioendothelioma. [Article in Japanese] Kyobu Geka 2009;62(3):223-6.

7. Chevreau C, Le Cesne A, Ray-Coquard I, Italiano A, Cioffi A, Isambert N, et al. Sorafenib in patients with progressive epithelioid hemangioendothelioma: a phase 2 study by the French Sarcoma Group (GSF/ GETO). Cancer 2013;119(14):2639-44.

8. Schattenberg T, Kam R, Klopp M, Herpel E, Schnabel PA, Mechtersheimer G, et al. Pulmonary epithelioid hemangioendothelioma: report of three cases. Surg Today 2008;38(9):844-9.

9. Weissferdt A, Moran CA. Primary vascular tumors of the lungs: a review. Ann Diagn Pathol 2010;14(4):296-308.
10. Weiss SW, Ishak KG, Dail DH, Sweet DE, Enzinger FM. Epithelioid hemangioendothelioma and related lesions. Semin Diagn Pathol 1986;3(4):259-87.

11. Dean PJ, Haggitt RC, O'Hara CJ. Malignant epithelioid hemangioendothelioma of the liver in young women. Relationship to oral contraceptive use. Am J Surg Pathol 1985;9(10):695-704.

12. Sicilian L, Warson F, Carrington CB, Hayes J, Gaensler EA. Intravascular bronchioloalveolar tumor (IV-BAT). Respiration 1983;44(5):387-94.

13. Amin RM, Hiroshima K, Kokubo T, Nishikawa M, Narita M, Kuroki M, et al. Risk factors and independent predictors of survival in patients with pulmonary epithelioid haemangioendothelioma. Review of the literature and a case report. Respirology 2006;11(6):818-25.

14. Ye B, Li W, Feng J, Shi JX, Chen Y, Han BH. Treatment of pulmonary epithelioid hemangioendothelioma with combination chemotherapy: Report of three cases and review of the literature. Oncol Lett 2013;5(5):1491-6.

15. Mizota A, Shitara K, Fukui T. Bevacizumab chemotherapy for pulmonary epithelioid hemangioendothelioma with severe dyspnea. J Thorac Oncol 2011;6(3):651-2. 